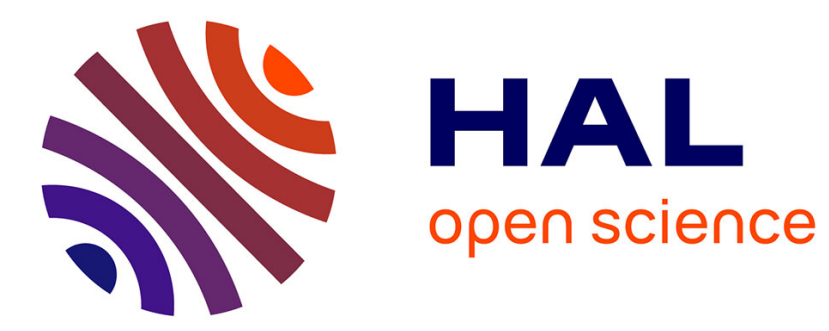

\title{
A mode-matching model for the impedance of perforated plate liners
}

Robin Billard, Gilles Tissot, Gwenael Gabard, Marc Versaevel, Jean-Philippe Groby

\section{- To cite this version:}

Robin Billard, Gilles Tissot, Gwenael Gabard, Marc Versaevel, Jean-Philippe Groby. A modematching model for the impedance of perforated plate liners. Aeroacoustics 2019 - 25th AIAA/CEAS Aeroacoustics Conference, May 2019, Delft, Netherlands. pp.1-12, 10.2514/6.2019-2702 . hal02335914

\section{HAL Id: hal-02335914 https://hal.inria.fr/hal-02335914}

Submitted on 28 Oct 2019

HAL is a multi-disciplinary open access archive for the deposit and dissemination of scientific research documents, whether they are published or not. The documents may come from teaching and research institutions in France or abroad, or from public or private research centers.
L'archive ouverte pluridisciplinaire HAL, est destinée au dépôt et à la diffusion de documents scientifiques de niveau recherche, publiés ou non, émanant des établissements d'enseignement et de recherche français ou étrangers, des laboratoires publics ou privés. 


\title{
A mode-matching model for the impedance of perforated plate liners
}

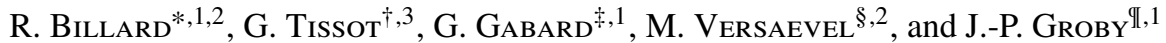 \\ ${ }^{1}$ Laboratoire d'Acoustique de l'Université du Mans, LAUM UMR CNRS 6613, av. Olivier Messiaen, 72085 Le Mans, \\ France \\ ${ }^{2}$ Safran Nacelles, Route du Pont VIII, 76700 Gonfreville-l'Orcher, France \\ ${ }^{3}$ INRIA Rennes Bretagne Atlantique, IRMAR - UMR CNRS 6625, av. Général Leclerc, 35042 Rennes, France
}

\begin{abstract}
A mode-matching model applicable to perforated liners in the linear regime is presented. It complements semi-empirical models not suitable for the high frequency regime, for liner featuring thick plate and micro perforated liner. The mentioned models are compared to measurements. From these comparisons, it appears that removing the short tube approximation is not enough to capture completely the losses in the linear regime. Further investigations have been carried out using a finite element method model based on compressible Linearised NavierStokes equations. This method showed to be in good agreement with experimental data. Thus, the dissipation due to viscosity was mapped around the aperture. This approach is supposed to provide a more precise understanding of the physical phenomenon of interest. Based on those results, the next step is to propose an upgraded mode-matching model computationally efficient that overtakes empirical models thanks to physical-based modeling.
\end{abstract}

\section{Introduction}

Perforated acoustics liners are a widespread technology used to reduce noise emissions from turbofan. Extensive

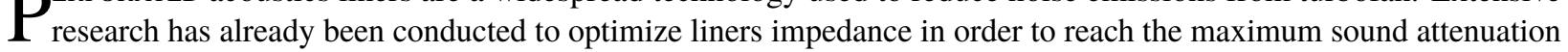
(see for instance Spillere [1] and Seo [2]). The linear physics phenomenon responsible for sound absorption are well known. However, the associated semi-empirical models such as Guess [3] or Maa [4], are applicable to a limited range of geometric configurations such as macro perforated liners. On the other hand, no reliable and time effective model appears to exist for micro perforated liners, presenting a percentage open area, named porosity, below $5 \%$ and a perforation diameter inferior to $1 \mathrm{~mm}$. Therefore, a Mode-Matching (MM) model based on Helmholtz equation is proposed. This approach allows to remove the short tube approximation, outlined further below. The model also includes the velocity profile through an aperture and the interaction effect between the perforations. This strategy is more comprehensive than the named semi-empirical models and is expected to be in better agreement with experimental data. A Finite Element Method model (FEM) based on compressible Linearised Navier-Stokes (LNS) equations is used as a subsequent comparison. As we shall see further, the results comparison tends to point out a missing dissipation mechanism in the current MM modeling. The corresponding phenomenon is highlighted through the post processing of FEM data, involving the analysis of the rate of dissipation due to viscosity near the perforation. The losses scheme highlighted on the aperture edges complete the existing visco-thermal modeling proposed by Zwikker \& Kosten [5] and Stinson [6] for cylindrical perforation. As a first step, the three models used in the study are detailed, beginning with Guess model, the mode-matching model and the numerical model. Thereafter, a comparison with measurements is presented. Finally, the dissipation analysis is described to provide further modeling perspectives.

Figure 1 introduce the modeled geometry composed of three cylindrical domains, namely the exterior $(I)$, the neck (II) and the cavity (III). A 2D axi-symmetrical problem is considered.

\footnotetext{
*Robin.Billard.Etu@univ-lemans.fr, Ph.D. Student

†illes.Tissot@inria.fr, Research fellow

$\doteqdot$ Gwenael.Gabard@univ-lemans.fr, Senior AIAA member

\$Marc.Versaevel@safrangroup.com, Acoustic \& lightning team manager

IJ Jean-Philippe.Groby@univ-lemans.fr
} 


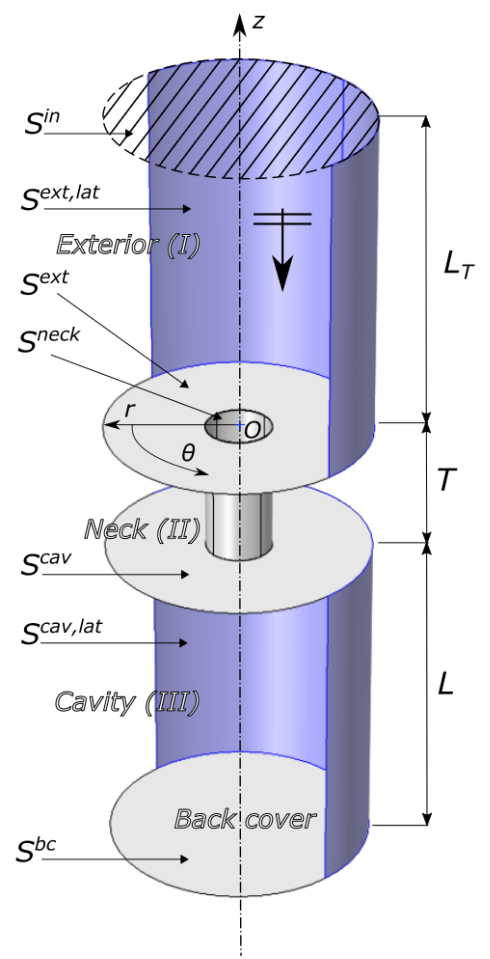

Figure 1 Scheme of the modeled geometry.

The radius of the exterior domain, the neck and the cavity domain are written, respectively $R^{\text {ext }}, R^{\text {neck }}$ and $R^{c a v}$. The plate porosity is noted $\sigma$. An $e^{\mathrm{j} \omega t}$ time convention is used.

\section{Semi empirical model}

Guess model [3] was chosen as a comparison basis for this study. The term accounting for viscosity is a common results derived by Zwikker \& Kosten [5] averaging the velocity $v$ across a tube and considering a linear pressure gradient along the tube, i.e. $\partial p / \partial z=\left(p_{\text {exit }}-p_{\text {entry }}\right) / T$. These assumptions are also known as the short tube approximation. Building this model on established results, in particular Morse \& Ingard [7] for radiation impedance and Ingard [8] for non linear impedance, Guess gives explicit expressions for the resistance and the reactance:

$$
\Theta=\mathfrak{R}\left(z_{v}\right)+\frac{2 \pi^{2}}{\sigma}\left(\frac{R^{n e c k}}{\lambda}\right)^{2} \quad \text { where } K^{2}=\frac{-\mathrm{j} \omega}{v},
$$

and,

$$
\chi=\mathfrak{J}\left(z_{v}\right)-\cot (k L)+\frac{\omega \delta}{\sigma \mathrm{c}_{0}},
$$

where $z_{v}$ is the non-dimensional impedance resulting from viscous and mass effects in the apertures:

$$
z_{v}=-\frac{\mathrm{j} \omega T}{\sigma \mathrm{c}_{0}}\left(\frac{2 \mathrm{~J}_{1}\left(K R^{\text {neck }}\right)}{K R^{\text {neck }} \mathrm{J}_{0}\left(K R^{\text {neck }}\right)}-1\right)^{-1} .
$$

$K$ is the Stokes wave number, $v$ is the kinematic viscosity, $\lambda$ is the wavelength, $k=\omega / c_{0}$ is the wave number, $\omega$ is angular frequency, $c_{0}$ is the sound speed, $\delta$ the end correction and $\mathrm{J}_{0}$ and $\mathrm{J}_{1}$ are the zeroth and first order Bessel functions, respectively, of the first kind (of complex argument). The $-\cot (k L)$ term corresponds to the cavity reactance. The present paper deals only with the linear regime, therefore, for the following comparisons to be consistent the non linear 
impedance is neglected. The end correction is defined as:

$$
\delta=\frac{16 R^{\text {neck }}}{3 \pi}(1-\sqrt{\sigma})\left(\frac{1+5 \cdot 10^{3}\left(\frac{\left|u_{0}\right|}{c_{0}}\right)^{2}}{1+10^{4}\left(\frac{\left|u_{0}\right|}{c_{0}}\right)^{2}}\right) .
$$

The $16 R^{\text {neck }} /(3 \pi)$ term is derived from Rayleigh's integral [9] and model the radiation effect. The factor $(1-\sqrt{\sigma})$ account for the interaction between multiple perforations. Finally the term featuring the acoustic velocity $u_{0}$ account for sound amplitude effects. Guess approximate equation (3) for the high and low frequency regime, namely for $\left|K R^{\text {neck }}>10\right|$ :

$$
z_{v} \simeq z_{v H}=\frac{\sqrt{2 v \omega} T^{\prime}}{\sigma c_{0} R^{n e c k}}+\mathrm{j}\left(\frac{\omega T}{\sigma c_{0}}+\frac{\sqrt{2 v \omega} T^{\prime}}{\sigma c_{0} R^{n e c k}}\right)
$$

and for $\left|K R^{\text {neck }}<1\right|$ :

$$
z_{v} \simeq z_{v L}=\frac{8 v T^{\prime}}{\sigma c_{0}\left(R^{\text {neck }}\right)^{2}}+\mathrm{j} \frac{4 \omega T}{3 \sigma c_{0}} .
$$

$T^{\prime}=T+2 R^{n e c k}$ is a corrected length accounting for the viscous effects occurring outside the perforation. It may be noted that equation (5) is suited for macro perforated configuration. In addition, approximations (5) and (6) are not valid for the micro perforated configurations studied further below, for which $1<\left|K R^{\text {neck }}\right|<10$. Lack of information is given to model the impedance in this range of parameters in Guess [3]. Therefore, in this set of parameters, the model is completed by implementing the following linear law:

$$
z_{v} \simeq z_{v M}=z_{v L}\left(\omega_{L}\right)+\frac{z_{v H\left(\omega_{H}\right)}-z_{v L\left(\omega_{L}\right)}}{\omega_{H}-\omega_{L}} \omega
$$

where $\omega_{H}=100 v /\left(R^{\text {neck }}\right)^{2}$ and $\omega_{H}=v /\left(R^{\text {neck }}\right)^{2}$.

\section{Mode-matching model}

The mode-matching model is based on three circular ducts, one interface between the exterior and the neck and one interface between the neck and the cavity. The exterior duct is semi-infinite and a unit plane wave with a normal incidence to the plate is considered. The propagation is modeled using Helmholtz equation in the absence of background flow and the following boundary conditions:

$$
\left\{\begin{array}{l}
\left(\Delta+k^{2}\right) p=0, \\
\nabla p \cdot \boldsymbol{n}=0 \text { on } S^{\text {ext }}, S^{c a v}, S^{\text {ext }, l a t}, S^{c a v, l a t} \text { and } S^{b c},
\end{array}\right.
$$

with $p$ the pressure field and $\boldsymbol{n}$ the ingoing unit vector. The corresponding solutions in the $(r, z)$ plane to Eq. 8) in the exterior, the neck and the cavity, respectively $I, I I$ and $I I I$, is of the form:

$$
p(r, z)^{h}=\sum_{m=0}^{\infty}\left(A_{m}^{h} e^{\mathrm{j} k_{z m}^{h} z}+B_{m}^{h} e^{-\mathrm{j} k_{z m}^{h} z}\right) \Phi_{m}^{h}, \text { with } h=I, I I, I I I .
$$

$m$ is the mode index and $\Phi_{m}$ is a shape function. The number of modes in domain $I$ and $I I I$ is truncated to $N$. When modes are added on both sides of the plate, the radiation impedance is accounted for naturally. The impact of this parameter will be discussed further below in section V. As we are making the plane wave hypothesis in the neck, one single mode is accounted for in domain II. The complex wave number used to model the attenuation in the neck reads:

$$
k_{z}^{I I}=\omega \sqrt{\rho(\omega) C(\omega)}
$$

where $\rho(\omega)$ is the equivalent complex density and $C(\omega)$ is the equivalent complex compressibility defined by Zwikker \& Kosten [5]. The velocities in domain $I$ and $I I I$ are determined using the Euler equation:

$$
v=\frac{\nabla p}{-\mathrm{j} \rho_{0} \omega}
$$


where $\rho_{0}$ is the air density. The velocity profile in the neck is taken into account in the MM model, the expression of $\boldsymbol{v}^{\text {neck }}$ projected on the axial unit vector $\boldsymbol{e}_{z}$, derived from Stinson [6], is:

$$
\boldsymbol{v}^{\text {neck }}(r, z) \cdot \boldsymbol{e}_{z}=\frac{\partial p}{\partial z} \frac{1}{\mathrm{j} \rho_{0} \omega}\left(\frac{\mathrm{J}_{0}(K r)}{\mathrm{J}_{0}\left(K R^{\text {neck }}\right)}-1\right) .
$$

The mode-matching method is implemented by enforcing the continuity of pressure and normal velocity at both interfaces [10]. When matching the exterior and the neck domain, the weak formulation for the pressure continuity can be expressed as:

$$
\forall n \in \mathbb{N} \quad \int_{0}^{S^{n e c k}} p^{n e c k} \Psi_{n}^{n e c k} \mathrm{~d} S^{n e c k}=\int_{0}^{S^{n e c k}} p^{e x t} \Psi_{n}^{n e c k} \mathrm{~d} S^{n e c k},
$$

with $p^{\text {neck }}$ the pressure field in the neck, $p^{\text {ext }}$ the pressure field in the exterior domain and $\Psi_{n}^{\text {neck }}$ the $n^{\text {th }}$ test function corresponding to the neck modal basis. The normal velocity continuity condition needs to respect $\boldsymbol{v}^{\text {ext }}(r, z=0) \cdot \boldsymbol{e}_{z}=$ $\boldsymbol{v}^{\text {neck }}(z=0) \cdot \boldsymbol{e}_{z}$ on $S^{\text {neck }}$ and $\boldsymbol{v}^{\text {ext }}(r, z=0) \cdot \boldsymbol{e}_{z}=0$ on $S^{\text {ext }} \backslash S^{\text {neck }}$. The corresponding weak formulation is expressed as follow:

$$
\forall l \in \mathbb{N} \quad \int_{0}^{S^{e x t}} \boldsymbol{v}^{e x t} \cdot \boldsymbol{e}_{z} \Psi_{l}^{e x t} \mathrm{~d} S^{e x t}=\int_{0}^{S^{n e c k}} \boldsymbol{v}^{\text {neck }} \cdot \boldsymbol{e}_{z} \Psi_{l}^{\text {ext }} \mathrm{d} S^{\text {neck }},
$$

with $v^{\text {ext }}$ the velocity field in the exterior domain, $v^{\text {neck }}$ the velocity field in the neck and $\Psi_{l}^{\text {neck }}$ the $l^{\text {th }}$ test function corresponding to the exterior modal basis. The same equations were used when matching the cavity and the neck domains. As mentionned above, a plane wave is implemented in the exterior domain. The ingoing plane wave with amplitude $A_{0}^{I}=1$ is implemented as:

$$
\nabla p \cdot \boldsymbol{n}=2 \mathrm{j} k A_{0}^{I} e^{-\mathrm{j} k L_{T}}+\mathrm{j} k p \text { on } S^{i n}
$$

Using the rigid boundary condition on the back cover, a linear system is composed, giving access to the modal amplitudes $A_{m}^{l}$ and $B_{m}^{l}$. Thus, the reflection coefficient is derived as $R_{M M}=B_{0}^{I} / A_{0}^{I}$. Finally, the impedance is determined using the following expression:

$$
z_{\omega}=\frac{1+R_{M M}}{1-R_{M M}}
$$

The stream line confinement driven by the periodic layout of the perforations (also known as the interaction effect) is modeled implicitly by adjusting the exterior radius to fit the plate porosity. Figure 2 illustrates this phenomenon.

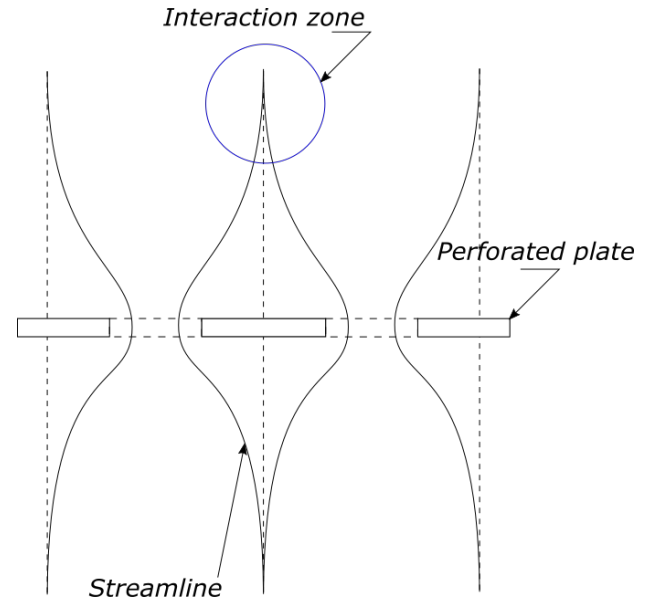

Figure 2 Scheme of the hole interaction effect.

This effect was also modeled by Melling [11] and Fok [12]. 


\section{Finite element method model}

A finite element method model based the linearised compressible Navier-Stokes equations is introduced. We are modelling the impedance of the geometry illustrated in Figure 1]. According to Roche [13], a 2D axi-symmetrical FEM domain is sufficient to capture correctly the physical phenomenon occurring in the linear regime without grazing flow.

In the following, $L_{r e f}$ is the physical distance which corresponds to a unitary distance in the mesh. We use $L_{r e f}=2 R^{c a v}$. The physical quantities are marked by $*$ and we define the non-dimensional as:

$$
x=\frac{x^{*}}{L_{\text {ref }}}, \quad \boldsymbol{u}=\frac{\boldsymbol{u}^{*}}{c_{0}}, \quad c=\frac{c^{*}}{c_{0}}, \quad \rho=\frac{\rho^{*}}{\rho_{0}}, \quad p=\frac{p^{*}}{\rho_{0} c_{0}^{2}}, \quad T_{e}=\frac{T_{e}^{*} c_{p}}{c_{0}^{2}}, \quad e=\frac{e^{*}}{c_{0}^{2}} .
$$

$\boldsymbol{u}$ is the velocity, $c$ is the sound speed, $\rho$ is the density, $p$ is the pressure, $T_{e}$ is the temperature, $c_{p}$ is the specific heat capacity at constant pressure and $e$ the specific internal energy. The following dimensionless numbers are introduced as well:

$$
\operatorname{Re}_{a}=\frac{\rho_{0} c_{0} L_{r e f}}{\mu_{0}}, \quad \operatorname{Pr}=\frac{c_{p} \mu_{0}}{k_{T_{e}}} .
$$

$R e_{a}$ is the acoustic Reynolds number, $P r$ is the Prandtl number, $\mu_{0}$ is the dynamic viscosity and $k_{T_{e}}$ the air thermal conductivity. The following governing equations derived from [14] are linearised around $\rho_{0}, \boldsymbol{u}_{0}, p_{0}, T_{e 0}$ and $\mu_{0}$ :

where $c_{v}$ is the specific heat capacity at constant volume. The deviatoric stress tensor $\tau$ can be expressed as:

$$
\tau=\frac{1}{R e_{a}}\left\{\left[\nabla \boldsymbol{u}+(\nabla \boldsymbol{u})^{\mathrm{T}}\right]+\left(\mu_{B}-\frac{2}{3} \mu\right) \nabla \cdot \boldsymbol{u} \mathbb{I}\right\},
$$

where $\mu_{B}=2 / 3$ is the non-dimensional bulk viscosity and $\mathbb{I}$ is identity matrix.

An adiabatic natural boundary condition $\nabla T_{e} \cdot \boldsymbol{n}=0$ with $\boldsymbol{n}$ the unit normal vector is enforced on every boundary. The free slip boundary conditions $\boldsymbol{u} \cdot \boldsymbol{n}=0$ are natural boundary conditions as well. Finally, an ingoing plane wave of amplitude $W$ is enforced using (15). The reflection coefficient is derived from (15) as:

$$
R_{F E M}=\frac{e^{-\mathrm{j} k L_{T}}}{W}\left(\bar{p}-W e^{-\mathrm{j} k L_{T}}\right),
$$

where $\bar{p}$ is the pressure averaged over the upper boundary of the domain. We will now focus on the numerical aspects of this model. The mesh is composed of Delaunay triangular elements, generated using Gmsh [15]. Figure 3 represents the mesh of configuration 3, of which the parameters are detailed in Table 2

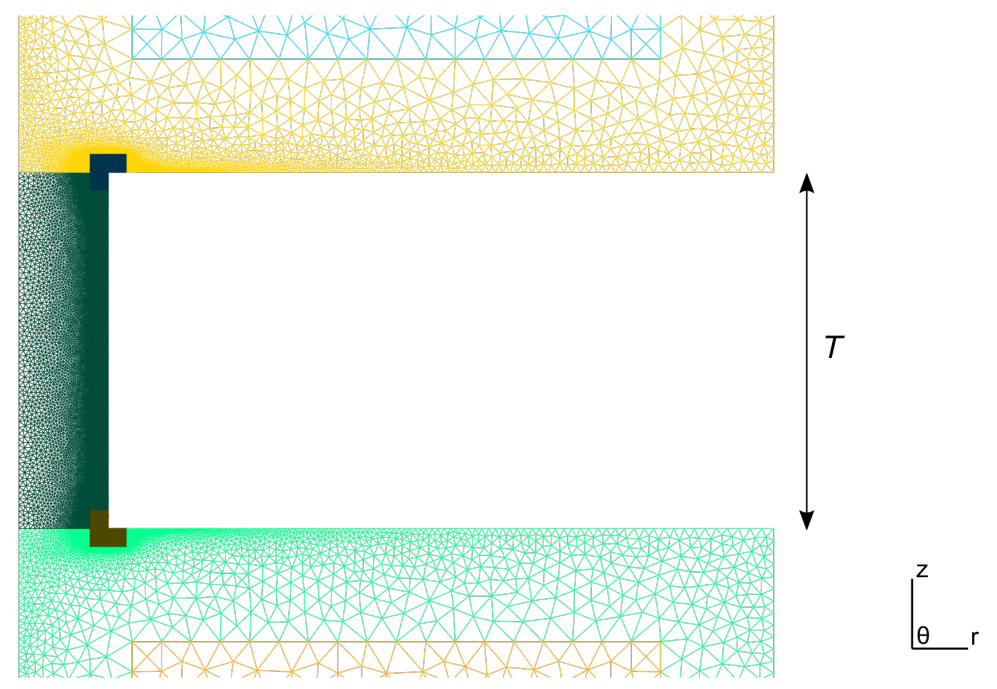

Figure 3 Mesh near the neck for configuration 3.

The mesh is refined on the domain boundaries and especially in the neck and its edges, i.e. where most of the dissipation mechanism are expected to occur. The numerical implementation is performed using GetFEM++ [16]. A 
second order Lagrange polynomial is chosen to interpolate the velocity $\boldsymbol{u}$. A first order polynomial is used for the density $\rho$ and the temperature $T_{e}$.

\section{Model comparison}

Four configurations have been chosen to perform a comparison between the three models described previously and experimental data. The corresponding parameters are summarized in Table 2. The measurements were carried out using a $29 \mathrm{~mm}$ diameter impedance tube with a $120 \mathrm{~dB}$ Sound Pressure Level (SPL). The frequency range is between $1000 \mathrm{~Hz}$ and $5000 \mathrm{~Hz}$ with a white noise source. The measured resonance frequency is noted $f_{\text {res }}$.

\begin{tabular}{|c|c|c|c|c|c|}
\hline Configuration & Porosity $\sigma(\%)$ & Radius $R^{\text {neck }}(\mathrm{mm})$ & Thickness $T(\mathrm{~mm})$ & Cavity height $L(\mathrm{~mm})$ & $f_{\text {res }}(\mathrm{Hz})$ \\
\hline \hline 1 & 6 & 0.8 & 1.5 & 19 & 1712 \\
2 & 14 & 0.8 & 2.1 & 19 & 2464 \\
3 & 1.4 & 0.15 & 0.6 & 19 & 1392 \\
4 & 4.2 & 0.15 & 0.6 & 19 & 2512 \\
\hline
\end{tabular}

Table 1 Perforated plate configurations.

The Helmholtz number $H_{e}$ is introduced as:

$$
H_{e}=\frac{L_{r e f}}{c_{0}} f^{*}
$$

where $f^{*}$ is the physical frequency. The upper graphs correspond to the resistance and the lower graphs to the plate reactance. In the following comparisons, "Guess" refers to Guess model using equation (3) and "Guess approximation" refers to Guess model using equations (6), (5) and (7) depending on the value of $\left|K R^{\text {neck }}\right|$. Results for macro perforated configurations are plotted on Figure 4 and 5 . In this case, $\left|K R^{\text {neck }}\right|$ is between 16 and 37. Thus, we're using Guess high frequency approximation, applicable to macro perforated liners. When no approximation are made on equation (3) and no corrected length is added to account for viscous effects at the entrances of the neck, the Guess and MM predictions are alike. When the Guess approximation is used, a good agreement is obtained between model and measurements, showing the impact of the corrected length $T^{\prime}$. The resistance is underestimated by the FEM model and by the MM model. The plate reactance is overestimated on the upper part of the Helmholtz number range by Guess approximation, Guess and the FEM. The MM slightly underestimates the plate reactance. The convergence of the MM method is shown by the correspondence of the impedance using 10 modes and 50 modes. 

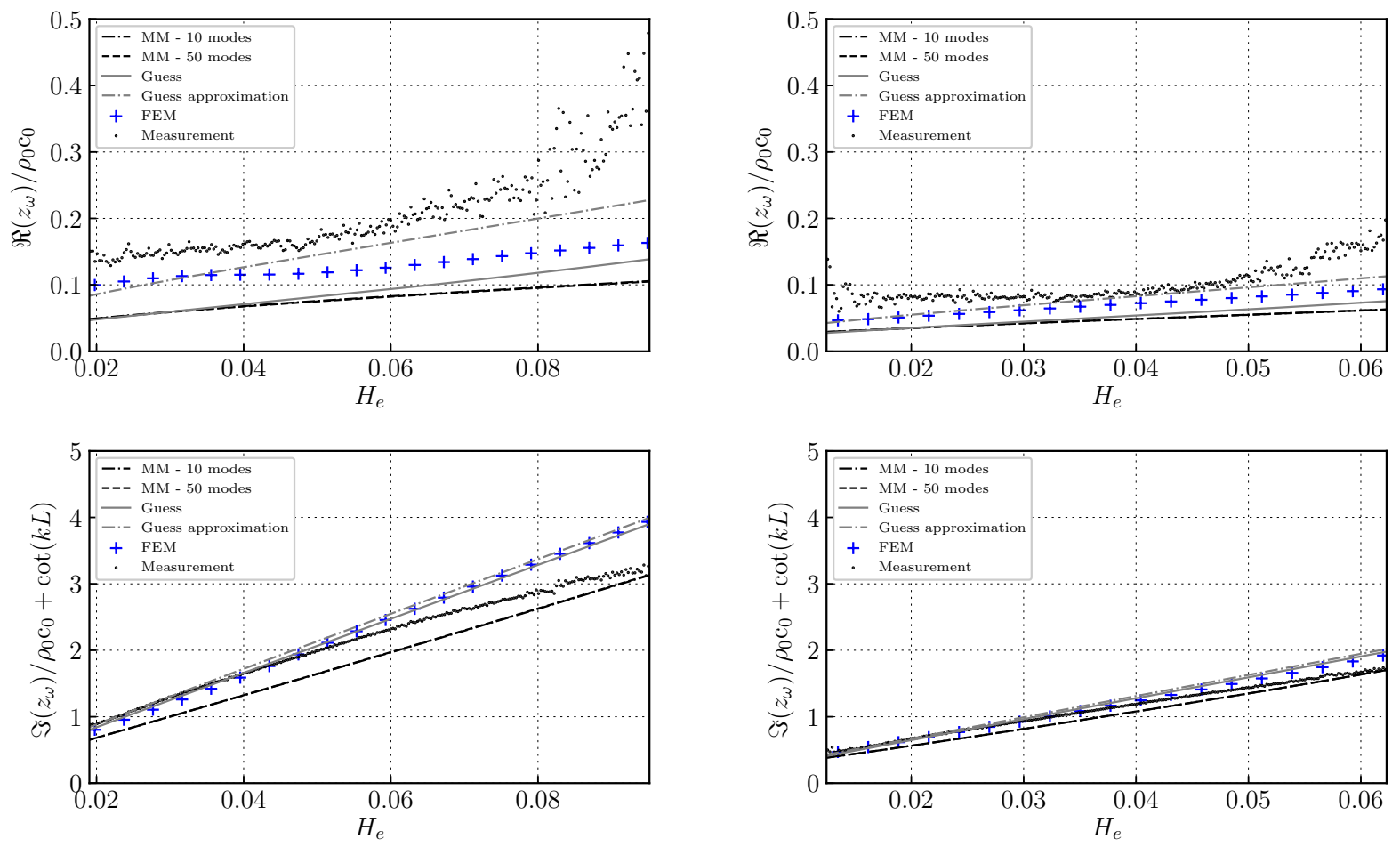
$\begin{array}{lll}\begin{array}{l}\text { Figure } 4 \\ \text { Impedance results for configuration 1, a }\end{array} & \begin{array}{l}\text { Figure } 5 \\ \text { Impedance results for configuration 2, a } \\ \text { macro perforated liner with a high porosity. }\end{array}\end{array}$

Results for micro perforated configurations are displayed on Figure 6 and $7 .\left|K R^{\text {neck }}\right|$ is now between 3 and 7. Once again, the mode-matching provides similar results to Guess without approximations and underestimates the resistance for both configurations. Guess approximation undervalues the resistance for configuration 3 while it is very accurate for configuration 4 , highlighting the validity limit of the corrected length $T^{\prime}$. The finite element method is correctly predicting the resistance for both configuration 3 and 4 . In the same way as the macro perforated configurations, the reactance is overestimated in the high half of the Helmholtz number range by the three models. 

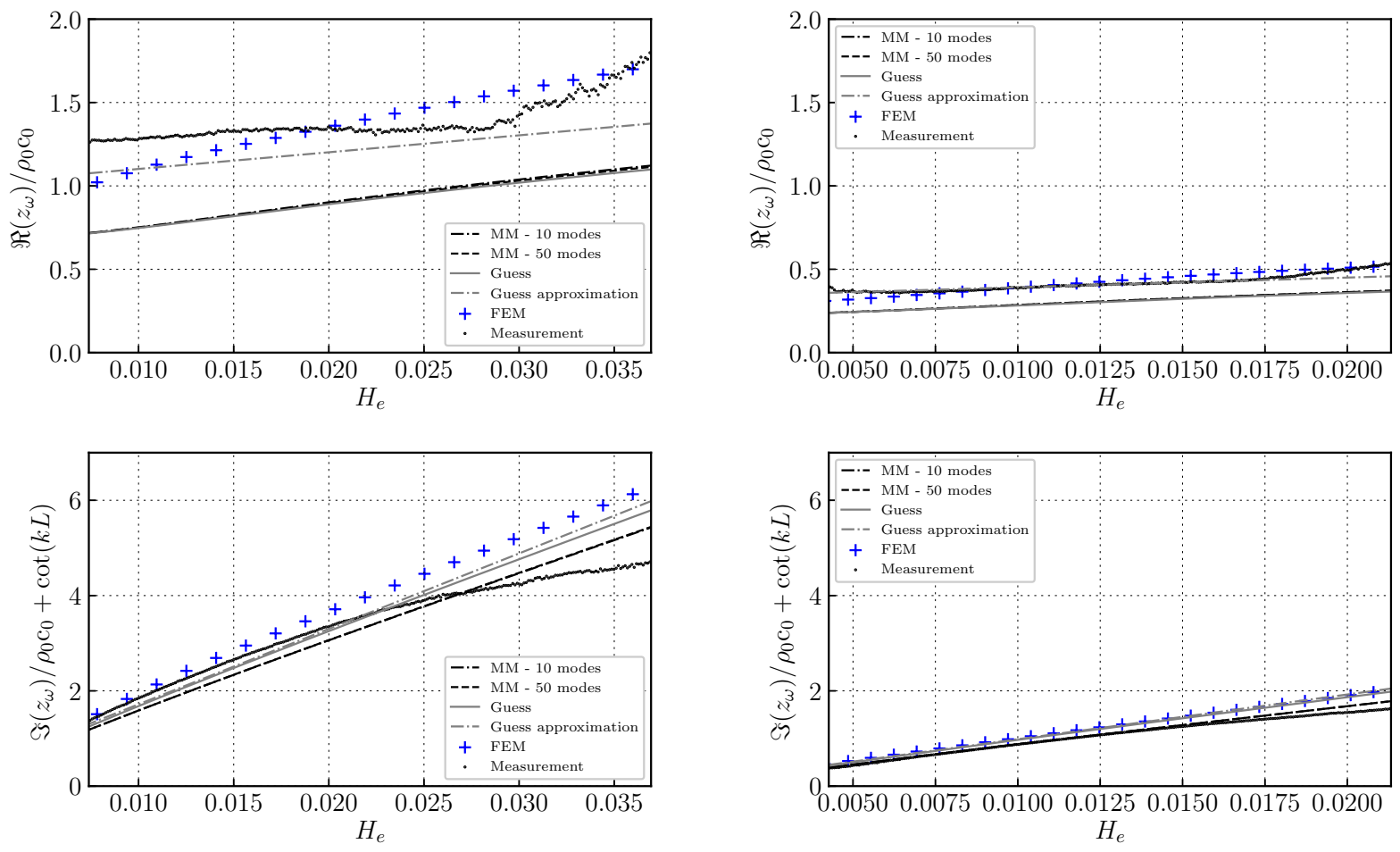

Figure 6 Impedance results for configuration 3, a mi- Figure 7 Impedance results for configuration 4, a micro perforated liner with a low porosity. cro perforated liner with a high porosity.

A dissipation mechanism seems to be absent from the MM model. Therefore, we investigate the velocity profile in the middle of the perforation and at its edges for configuration 3 . We compare the axial velocity obtained with the mode-matching and the norm of the velocity obtained with the FEM. This comparison is carried out for two locations, namely at $z=0$ and $z=-T / 2$. This is conducted for a 10 modes computation on Figure 8 and a 50 modes computation on Figure 9. The profiles are consistent with Stinson [6] narrow tube classification, i.e. when the viscous boundary layer is large compared to the tube radius. The profiles are plotted at $1270 \mathrm{~Hz}$ near the measured resonance frequency, i.e. $1392 \mathrm{~Hz}$. 


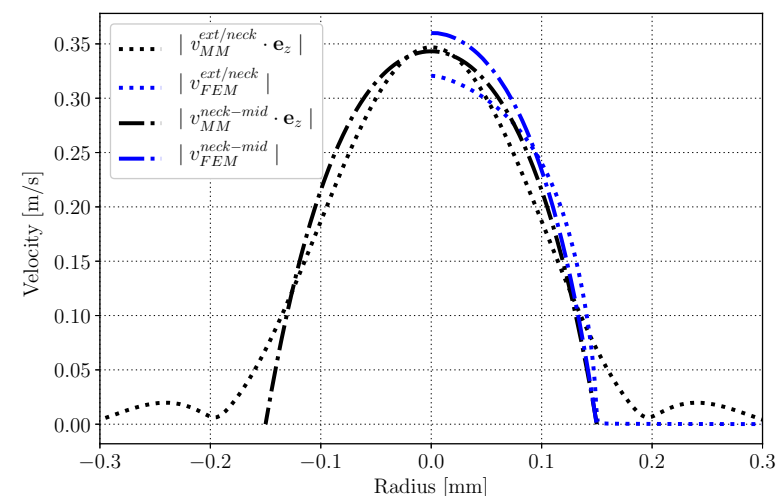

Figure 8 Velocity profile obtained through mode matching (10 modes) and FEM at the interface exterior/neck $(z=0)$ and in the middle of the neck $(z=-T / 2)$.

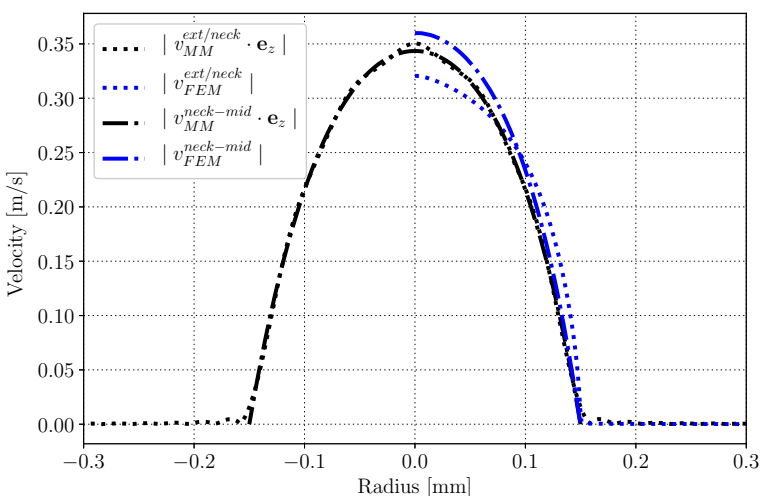

Figure 9 Velocity profile obtained through mode matching (50 modes) and FEM at the interface exterior/neck $(z=0)$ and in the middle of the neck $(z=-T / 2)$.

The agreement between mode matching and FEM is globally good except near the discontinuity due to the corner of the perforation at $r=0.15 \mathrm{~mm}$. This zone requires a certain number of modes to be described correctly by the MM model. Even though oscillations are still visible in the 50 modes case, their impact on impedance results appeared to be negligible when observing Figure 4 A velocity gradient is noticeable between $z=0$ and $z=-T / 2$ in the FEM. Indeed, at $r=0$, we have $\left|v_{F E M}^{\text {next } / \text { neck }}\right|=0.36 \mathrm{~m} / \mathrm{s}$ and $\left|\boldsymbol{v}_{F E M}^{\text {neck-mid }}\right|=0.32 \mathrm{~m} / \mathrm{s}$. Such a velocity gradient is not observed in the mode-matching. Furthermore, it may be noted that Guess neglects this effect when using the short tube approximation.

\section{Dissipation rate}

The dissipation mechanism about the neck appear to be modeled incompletely by the mode matching. With the intention of identifying the remaining losses, the rate of dissipation per unit mass due to viscosity is estimated through the previous FEM. With our choice of $\mu_{B}$, it's dimensionless expression is derived from Batchelor [17] as:

$$
D(\boldsymbol{u})=\frac{2}{R e_{a}}\left\{\frac{1}{4}\left[\nabla \boldsymbol{u}+(\nabla \boldsymbol{u})^{\mathrm{T}}\right]:\left[\nabla \boldsymbol{u}+(\nabla \boldsymbol{u})^{\mathrm{T}}\right]-\frac{1}{3} \operatorname{Tr}(\nabla \boldsymbol{u})^{2}\right\},
$$

where $\operatorname{Tr}$ is the trace. Figures 10 and 11 are a representation of $D(\boldsymbol{u})$ through a colormap focusing on the upper corner and the neck at $1270 \mathrm{~Hz}$ near the measured resonance frequency, i.e. $1392 \mathrm{~Hz}$.

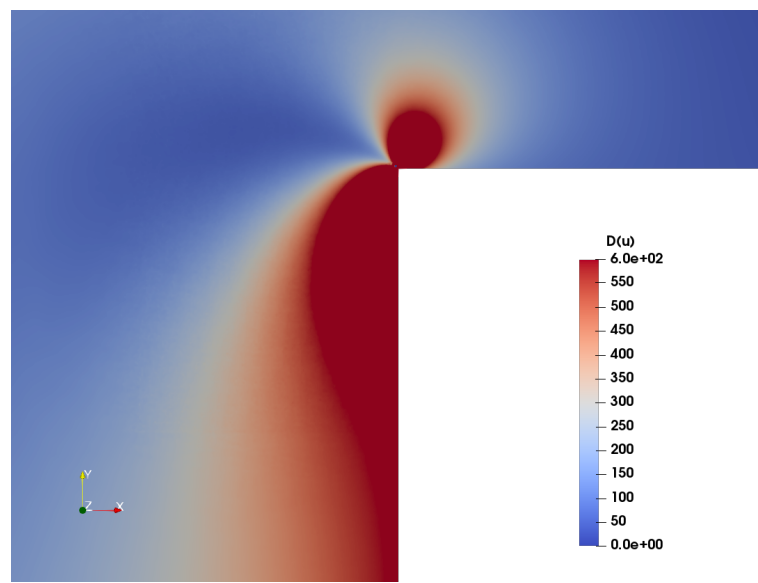

Figure 10 Dimensionless dissipation rate near the corner for configuration 1.

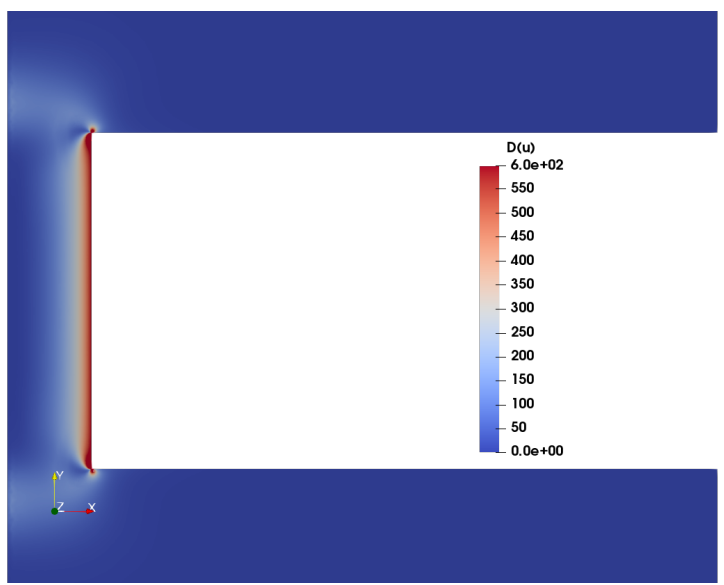

Figure 11 Dimensionless dissipation rate near the neck for configuration 1 . 
The dissipation resulting from the acoustic boundary layer in the neck is clearly observable, and is modelled by MM through the Zwicker \& Kosten losses. The dissipation mechanism in the corner zone seems to be significant though it is not explicitly accounted for in any model. On the other hand, the length correction proposed by Guess to account for viscous effects outside the perforation appears to be accurate, and to implicitly account for the named corner effect. A similar phenomenon is reported by Roche [18]. This cannot be taken into account in the MM plane wave modeling in the neck. That may explain the mismatch in terms of resistance between MM with both FEM and Guess.

We now integrate the dissipation rate in four different domain for all four configurations near their respective resonances frequencies. Figure 12 map the mentioned zones, situated around the aperture.

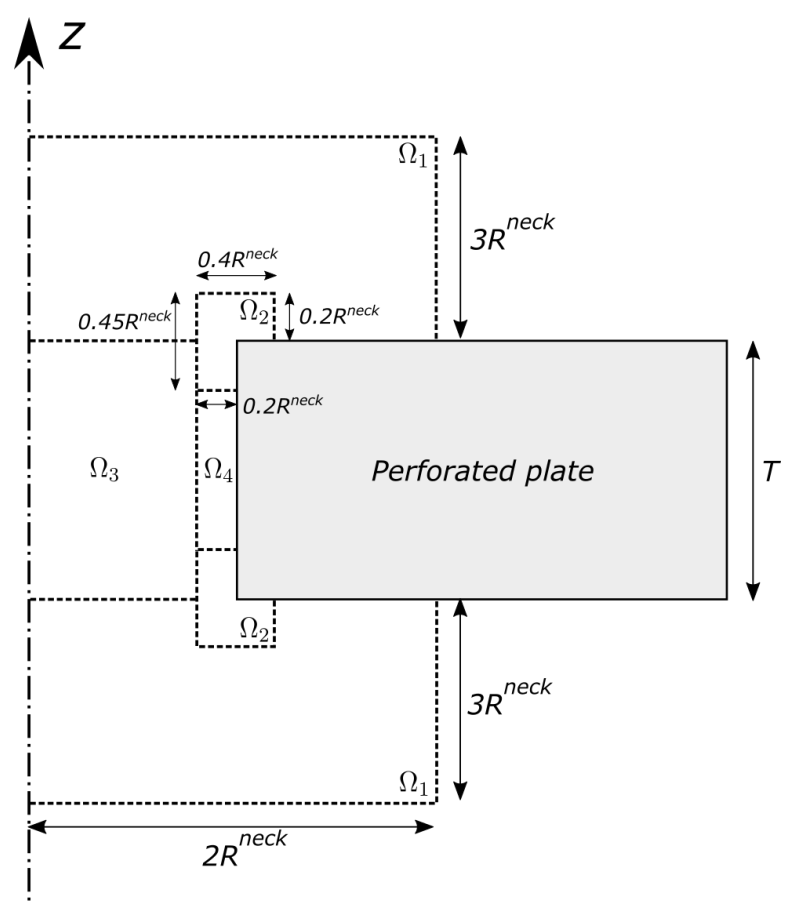

Figure 12 Scheme of the dissipation zones.

$\Omega_{1}$ corresponds to a domain surrounding the aperture. $\Omega_{2}$ stands for the edges of the neck, where the dissipation mechanism displayed on Figure 10 is taking place. Finally, $\Omega_{3}$ coincide with the neck region and $\Omega_{4}$ corresponds to a region close to the wall, where a significant part of the losses occurs. For a better interpretation of the dissipation mechanism in the perforation, we introduce the acoustic viscous boundary layer thickness $\delta_{V}$ (see Bruneau [19] for reference), which can be written as follows:

$$
\delta_{V}=\sqrt{\frac{2 v}{\omega}}
$$

Table 2 presents the relative contributions from each domain as a percentage of the total viscous losses in the FEM domain. For each configuration, the remaining losses apart from the four domains, are inferior or equal to $0.5 \%$.

\begin{tabular}{|c|c|c|c|c|c|c|c|}
\hline Configuration & $\Omega_{1}$ & $\Omega_{2}$ & $\Omega_{3}$ & $\Omega_{4}$ & Frequency & $2 R^{\text {neck }} / T$ & $\delta_{V} / R^{\text {neck }}$ \\
\hline \hline 1 & $14.5 \%$ & $46.7 \%$ & $3.7 \%$ & $34.7 \%$ & $1870 \mathrm{~Hz}$ & 0.94 & 0.03 \\
2 & $7.7 \%$ & $38.6 \%$ & $1.0 \%$ & $52.5 \%$ & $2379 \mathrm{~Hz}$ & 0.76 & 0.03 \\
3 & $25.3 \%$ & $8.7 \%$ & $30.2 \%$ & $35.2 \%$ & $1270 \mathrm{~Hz}$ & 0.5 & 0.41 \\
4 & $25.3 \%$ & $10.5 \%$ & $25.6 \%$ & $38.2 \%$ & $2275 \mathrm{~Hz}$ & 0.5 & 0.31 \\
\hline
\end{tabular}

Table 2 Dissipation rate contributions from the four domains. 
The corners contributions is quite low when considering the micro perforated configurations $3 \& 4$, for which the $2 R^{\text {neck }} / T$ ratio is low. As the boundary layer thickness is large compared to the aperture radius, the dissipation is significant both in domain $\Omega_{3}$ and $\Omega_{4}$. When $2 R^{\text {neck }} / T$ is closer to 1 , i.e. for macro perforated configurations $1 \& 2$, the corners contributions is much more significant. In that case, the boundary layer thickness is small compared to the aperture radius, consistent with the fact that the dissipation is substantial in domain $\Omega_{4}$ and minor in domain $\Omega_{3}$. Finally, the dissipation in $\Omega_{1}$ constitutes a quarter of the losses of the micro perforated configurations. This value drop to $14.5 \%$ and $7.7 \%$ for macro perforated configurations, showing the impact of the porosity on the dissipation surrounding the aperture.

\section{Perspectives}

The edges effects appear to be more pronounced for macro perforated configurations than for micro perforated configurations. Therefore, the Zwikker \& Kosten theory seem to be more adapted for the micro perforated case. In order to properly account for the dissipation on the corners, the modeling effort might need to focus on high order viscous modes in the neck of circular cross section. Alternatively, the losses can be completed using the empirical length corrections currently available in the literature, which is a reliable way to model the impedance of liners in a given range of parameters. However, this type of corrections are not adapted yet to unconventional liners, such as micro perforated liners or conical apertures liners. In recent years, theoretical studies have been conducted by Bendali et al. [20] and Laurens et al. [21] providing the Rayleigh's conductivity for the named non standard configurations in the linear regime. Regarding conical perforations, Honzík et al. [22] has proposed a transfer matrix accounting for the visco-thermal losses in the neck. In a forthcoming work, it is intended to compare these theoretical results with measurements.

\section{Acknowledgement}

The authors gratefully acknowledge the support from ANR Chaire industrielle MACIA (ANR-16-CHIN-0002). This study is done in the context of a CIFRE Ph.D. Thesis financed by the ANRT and Safran.

\section{References}

[1] Spillere, A. M., Medeiros, A. A., and Cordioli, J. A., "An improved impedance eduction technique based on impedance models and the mode matching method," Applied Acoustics, Vol. 129, Jan. 2018, pp. 322-334.

[2] Seo, S.-H., Kim, Y.-H., and Kim, K.-J., "Design of silencer using resonator arrays with high sound pressure and grazing flow," Applied Acoustics, Vol. 138, Sept. 2018, pp. 188-198.

[3] Guess, A. W., "Calculation of perforated plate liner parameters from specified acoustic resistance and reactance," Journal of Sound and Vibration, Vol. 40(1), 1975, pp. 119-137.

[4] Maa, D.-Y., "Potential of microperforated panel absorber," Acoustical Society of America, 1998, pp. 6.

[5] Zwikker, C. and Kosten, C. W., Sound absorbing materials, Elsevier, New York, 1949.

[6] Stinson, M. R., "The propagation of plane sound waves in narrow and wide circular tubes, and generalization to uniform tubes of arbitrary cross-sectional shape," The Journal of the Acoustical Society of America, Vol. 89, No. 2, Feb. 1991, pp. 550-558.

[7] Morse, P. M. and Ingard, K. U., Theoretical Acoustics, McGraw-Hill Book Company, New York, 1968.

[8] Ingard, U., "Absorption Characteristics of Nonlinear Acoustic Resonators," The Journal of the Acoustical Society of America, Vol. 44, No. 4, Oct. 1968, pp. 1155-1156.

[9] Pierce, A. D., Acoustics: An Introduction to its Physical Principles and Applications, McGraw-Hill Book Company, New York, 1981.

[10] Sergent, P., Optimisation géométrique du contrôle actif dans les gaines de ventilation, Ph.D. thesis, École Nationale des Ponts et Chaussées, 1996.

[11] Melling, T., "The acoustic impendance of perforates at medium and high sound pressure levels," Journal of Sound and Vibration, Vol. 29, No. 1, July 1973, pp. 1-65.

[12] Fok, V. A., "Theoretical research of the conductivity of a circular aperture in a partition across a pipe," Doklady akademii nauk SSSR, Vol. 31, No. 9, 1941, pp. 875-82. 
[13] Roche, J.-M., Leylekian, L., Delattre, G., and Vuillot, F., "Aircraft Fan Noise Absorption: DNS of the Acoustic Dissipation of Resonant Liners," 15th AIAA/CEAS Aeroacoustics Conference (30th AIAA Aeroacoustics Conference), American Institute of Aeronautics and Astronautics, Miami, Florida, May 2009.

[14] Wendt, J. F. and Anderson, J. D., Computational Fluid Dynamics - An introduction: With contributions by John D. Anderson, Springer, Berlin, Heidelberg, 2009.

[15] Geuzaine, C. and Remacle, J.-F., "Gmsh: a three-dimensional finite element mesh generator with built-in pre- and post-processing facilities," International Journal for Numerical Methods in Engineering, Vol. 79, No. 11, 2009, pp. 1309-1331.

[16] Renard, Y. and Pommier, J., “GetFEM++. An Open Source Finite Element Library,” 2017.

[17] Batchelor, G. K., An introduction to fluid dynamics, Cambridge mathematical library, Cambridge Univ. Press, Cambridge, 1st ed., 2010, OCLC: 838184093.

[18] Roche, J.-M., Simulation numérique de l'absorption acoustique de matériaux résonnants en présence d'écoulement., Ph.D. thesis, Université du Mans, 2011.

[19] Bruneau, M. and Scelo, T., Fundamentals of acoustics, ISTE, London, 2006, OCLC: 255489082.

[20] Bendali, A., Fares, M., Piot, E., and Tordeux, S., "Mathematical Justification of the Rayleigh Conductivity Model for Perforated Plates in Acoustics," SIAM Journal on Applied Mathematics, Vol. 73, No. 1, Jan. 2013, pp. 438-459.

[21] Laurens, S., Piot, E., Bendali, A., Fares, M., and Tordeux, S., "Effective conditions for the reflection of an acoustic wave by low-porosity perforated plates," Journal of Fluid Mechanics, Vol. 743, March 2014, pp. 448-480.

[22] Honzík, P., Fohr, F., and Bruneau, M., “Transfer function of small convergent-divergent (C-D) nozzles and opposite (D-C) devices filled with thermo-viscous fluid at rest," Applied Acoustics, Vol. 99, Dec. 2015, pp. 118-124. 